\title{
Perspectives on immune checkpoint ligands: expression, regulation, and clinical implications
}

\author{
Jihyun Moon ${ }^{1,2, \#}$, Yoo Min $\mathrm{Oh}^{1,2, \#} \mathcal{E}$ Sang-Jun $\mathrm{Ha}^{1,2, *}$ \\ ${ }^{1}$ Department of Biochemistry, College of Life Science \& Biotechnology, Yonsei University, Seoul 03722, ${ }^{2}$ Brain Korea 21 (BK21) FOUR \\ Program, Yonsei Education \& Research Center for Biosystems, Yonsei University, Seoul 03722, Korea
}

In the tumor microenvironment, immune checkpoint ligands (ICLs) must be expressed in order to trigger the inhibitory signal via immune checkpoint receptors (ICRs). Although ICL expression frequently occurs in a manner intrinsic to tumor cells, extrinsic factors derived from the tumor microenvironment can fine-tune ICL expression by tumor cells or prompt non-tumor cells, including immune cells. Considering the extensive interaction between $\mathrm{T}$ cells and other immune cells within the tumor microenvironment, ICL expression on immune cells can be as significant as that of ICLs on tumor cells in promoting antitumor immune responses. Here, we introduce various regulators known to induce or suppress ICL expression in either tumor cells or immune cells, and concise mechanisms relevant to their induction. Finally, we focus on the clinical significance of understanding the mechanisms of ICLs for an optimized immunotherapy for individual cancer patients. [BMB Reports 2021; 54(8): 403-412]

\section{INTRODUCTION}

In cancer, it is the cytolytic action of cytotoxic lymphocytes that the immune system mainly elicits to restrain disease progression. However, CD8 T cells in the tumor microenvironment frequently undergo 'exhaustion', which is a distinct developmental process, because they are faced with sustained antigenic stimulation. Exhausted CD8 T cells persist but gradually lose their effector function, cytotoxicity, and proliferative capacity, leading to incompetent immunosurveillance.

Exhausted CD8 T cells typically express a panoply of inhibitory immune checkpoint receptors (ICRs), which are triggered

*Corresponding author. Tel: +82-2-2123-2696; Fax: +82-2-362-9897; E-mail: sjha@yonsei.ac.kr

${ }^{\text {"}}$ These authors contributed equally to this work.

https://doi.org/10.5483/BMBRep.2021.54.8.054

Received 23 March 2021, Revised 3 May 2021, Accepted 14 May 2021

Keywords: Immune checkpoint ligand, Immune checkpoint receptor, Immunotherapy, Tumor microenvironment by cognate ligands to regulate T-cell response via a downstream signaling pathway. For instance, programmed cell death 1 (PD-1, CD279), one of the notable ICRs, is expressed on exhausted CD8 T cells. Upon being bound with programmed cell deathligand 1 (PD-L1, B7-H1, CD274), PD-1 counters either CD28 signaling or a T-cell receptor (TCR) by activating $\mathrm{SH} 2$ domaincontaining protein tyrosine phosphatase-2 (SHP-2) and renders the T cells hypofunctional in many ways (1). Therefore, in exhausted CD8 T cells, blocking the interactions between ICRs and immune checkpoint ligands (ICLs) is a plausible strategy for de-repressing CD8 T cells. Indeed, immune checkpoint blockade therapy has demonstrated clinical efficacy against various types of tumors (2). Multiple immune checkpoint pathways other than PD-1/PD-L1 have been investigated (3), and the pathways vary from patient to patient because of the heterogeneity of the tumor microenvironment. Since most ICRs were co-expressed as part of a larger co-inhibitory gene program (4), the 'functional' inhibitory pathways are determined by the expression of ICLs. Therefore, it is essential to understand which factors contribute to the inter-patient differences in ICL expressions. In this review, we summarize various factors that modulate the expression of ICLs within the tumor microenvironment (Table 1)

\section{PD-1 LIGANDS}

The promising outcome of therapies targeting the PD-1 axis has highlighted the need to elucidate the molecular regulation of its ligands. PD-1-mediated T-cell inhibition can be attributed to both of two well-known ligands of PD-1, which are PD-L1 and programmed cell death-ligand 2 (PD-L2, B7-DC, CD273) (Figs. 1 and 2).

\section{PD-L1}

Given that antibodies against PD-L1 have shown an efficacy similar to that of the antibodies against PD-1, which can block interactions with both PD-L1 and PD-L2, PD-L1 is a more dominant ligand of PD-1 than is PD-L2. PD-L1 expression by tumor cells often occurs during malignant transformation and without ongoing immune response (5). However, apart from intrinsic factors, which give rise to constitutive expression of PD-L1 in tumor cells, extrinsic factors within the tumor micro-

ISSN: 1976-670X (electronic edition)

Copyright (C) 2021 by the The Korean Society for Biochemistry and Molecular Biology

c) This is an open-access article distributed under the terms of the Creative Commons Attribution Non-Commercial License (http://creativecommons.org/licenses/by-nc/4.0) which permits unrestricted non-commercial use, distribution, and reproduction in any medium, provided the original work is properly cited. 
Regulation of immune checkpoint ligand expression and its clinical application Jihyun Moon, et al.

Table 1. Overview of multiple immune check point ligand regulators

\begin{tabular}{|c|c|c|c|c|}
\hline \multirow{2}{*}{ Receptor } & \multirow{2}{*}{ Ligand } & \multirow{2}{*}{ Regulator } & \multicolumn{2}{|c|}{ Expression } \\
\hline & & & Tumor cell & Immune cell \\
\hline \multirow[t]{24}{*}{ PD-1 } & PD-L1 & $\mathrm{IFN} \gamma$ & Multiple tumor cell $(10,11)$ & $\begin{array}{l}\text { Dendritic cell }(24,25), \text { neutrophil }(27), \\
\text { monocyte/macrophage }(24,26)\end{array}$ \\
\hline & & IFN $\beta$ & H melanoma (11) & Dendritic cell (28), neutrophil (27) \\
\hline & & TNF $\alpha$ & $\begin{array}{l}\text { H breast cancer (12), H prostate/ } \\
\text { colon cancer (13), H renal cell carcinoma (14) }\end{array}$ & Dendritic cell (29), monocyte/macrophage $(30,31)$ \\
\hline & & IL-6 & H prostate cancer (15), H lung cancer (16) & Dendritic cell (29), monocyte/macrophage (32) \\
\hline & & TLR3 & H neuroblastoma $(21)$ & Dendritic cell $(40)$ \\
\hline & & TLR4 & H bladder cancer (20) & Dendritic cell $(43)$, monocyte/macrophage $(41,42)$ \\
\hline & & IL-4 & $\mathrm{H}$ renal cell carcinoma (14) & - \\
\hline & & IL-27 & H ovarian cancer (18) & - \\
\hline & & TGF $\beta$ & H lung cancer (19) & - \\
\hline & & IL-10 & - & Monocyte $(33,35,36)$ \\
\hline & & IL-17 & - & Monocyte/macrophage (33) \\
\hline & & IL-1 $\beta$ & - & Dendritic cell (29) \\
\hline & & PGE2 & - & Monocyte/macrophage (44) \\
\hline & & Nutrient deprivation & Multiple tumor cell $(6,7)$ & - \\
\hline & & Metabolite accumulation & H lung cancer (8) & - \\
\hline & & Hypoxia & Multiple tumor cell (9) & - \\
\hline & & Oncogenic signaling & Multiple tumor cell (5) & - \\
\hline & & microRNA & Multiple tumor cell (5) & - \\
\hline & PD-L2 & $\mathrm{IL}-4$ & H esophageal adenocarcinoma (49) & $\begin{array}{l}\text { Dendritic cell (51), } \\
\text { monocyte/macrophage }(42,51,52)\end{array}$ \\
\hline & & IFN $\gamma$ & $\begin{array}{l}\text { H melanoma (11), H lung cancer (19), } \\
\text { H colorectal cancer (47), H brain tumor (48) }\end{array}$ & Monocyte/macrophage $(24,36,50)$ \\
\hline & & IFN $\beta$ & H melanoma (11) & \\
\hline & & IL-13 & H esophageal adenocarcinoma (49) & \\
\hline & & IL-2/IL-15/IL-21 & - & Monocyte/macrophage (54) \\
\hline & & GM-CSF & - & Dendritic cell/macrophage (53) \\
\hline \multirow[t]{6}{*}{ TIGIT } & PVR & RAS/RAF/MEK/ERK & Fibroblast (60) & - \\
\hline & & DNA damage & H melanoma $(63,64)$ & - \\
\hline & & IKZF-1/3 & H melanoma (65) & - \\
\hline & & ER stress & H hepatoma (66) & - \\
\hline & & $\begin{array}{l}\text { SUMO-conjugating } \\
\text { enzyme UBC9 }\end{array}$ & H melanoma (67) & - \\
\hline & & TLR1/2/3/4/7/8/9 & - & Dendritic cell/macrophage (69) \\
\hline \multirow[t]{4}{*}{ TIM3 } & Galectin-9 & IFN $\gamma$ & - & Monocytes/gMDSCs $(74,79)$ \\
\hline & & IFN $\beta$ & H leukemia/M colon cancer (73) & - \\
\hline & & microRNA & H chondrosarcoma (80), liver cancer (81) & - \\
\hline & & DNMT3A & H cervical cancer (82) & - \\
\hline \multirow[t]{5}{*}{ LAG3 } & $\mathrm{MHCll}$ & $\mathrm{IFN} \gamma$ & H osteosarcoma/H melanoma (84) & - \\
\hline & Galectin-3 & NF-kB & H leukemia (89) & Macrophage (90) \\
\hline & LSECtin & IL-6/IL-10 & $\mathrm{H} / \mathrm{M}$ melanoma (86) & 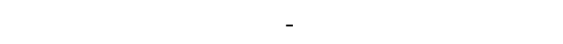 \\
\hline & & IL-4 & - & Dendritic cell (91) \\
\hline & FGL-1 & IL-6 & H hepatocellular carcinoma (93) & - \\
\hline
\end{tabular}

$M$, mouse; $\mathrm{H}$, human.

environment can contribute to PD-L1 expression. Many of the factors in the tumor vicinity, such as nutrient deprivation (6, 7), metabolite accumulation (8), and hypoxia (9), are observed in common in different types of tumors, concomitantly causing PD-L1 upregulation. Further, an inflammatory tumor microenvironment provides the assorted factors that adjust PD-L1 


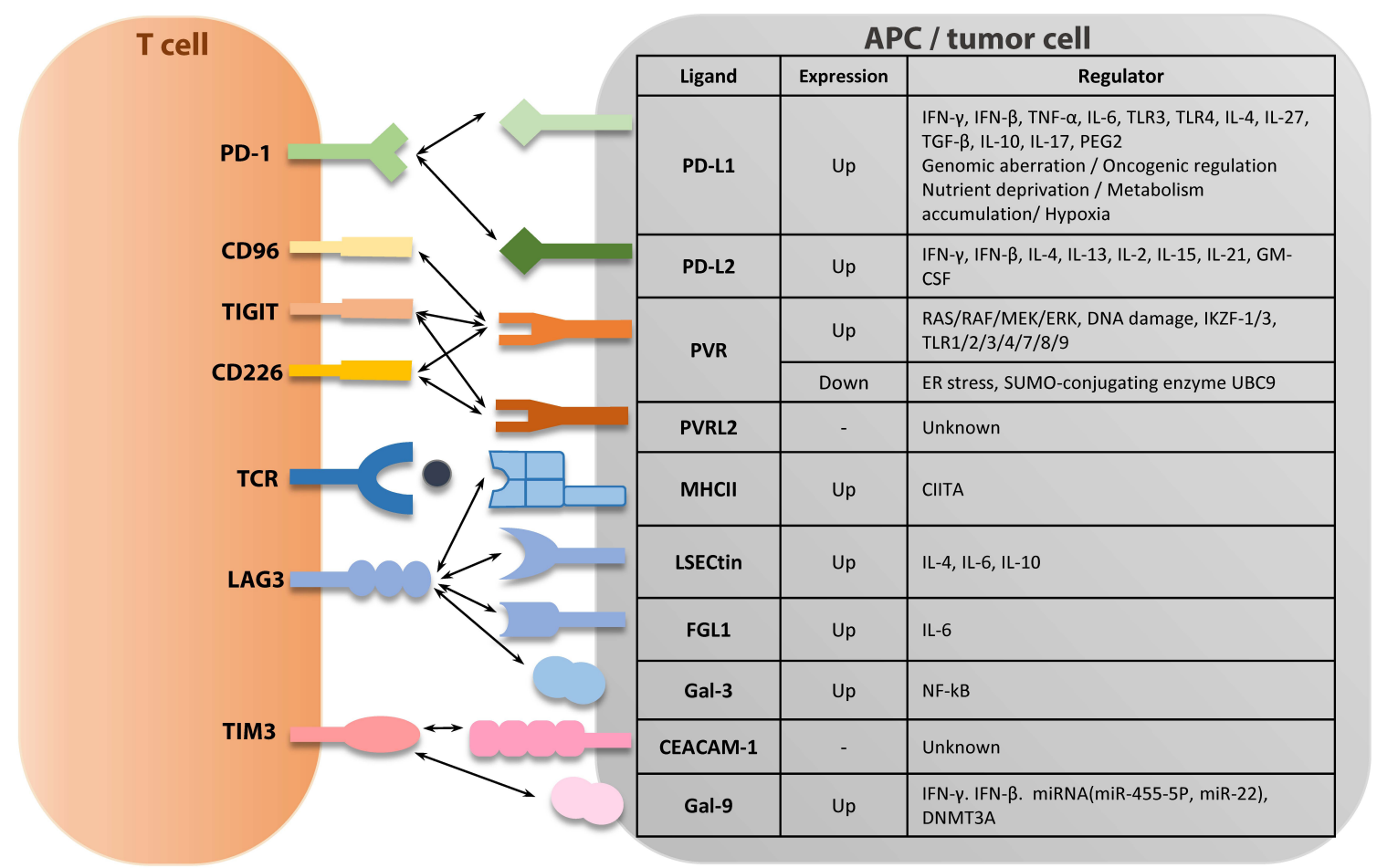

Fig. 1. Multiple immune checkpoint receptor-ligand interactions between $\mathrm{T}$ cells and APCs or tumor cells. The regulators of each immune checkpoint ligand are indicated on the right side (Up, positive regulator; Down, negative regulator).

expression. IFN- $\gamma$, primarily secreted by effector $\mathrm{T}$ cells and NK cells, is the most potent inducer of PD-L1 for various tumor-cell lines (10); hence PD-L1 expression in tumor cells may reflect concurrent T-cell responses. Mechanistically, in human melanoma cell lines, IFN- $\gamma$-induced upregulation of PD-L1 is mediated by JAK1/2 - STAT1 activation and, eventually, direct binding of Interferon Regulatory Factor 1 (IRF1) to PD-L1 promoter (11). Garcia-Diaz et al. also clarify that IFN- $\alpha$ and IFN- $\beta$ induce PD-L1 expression, but to a lesser extent than does IFN- $\gamma$ (11). Besides IFN, other inflammatory mediators regulate PD-L1 expression as well. For example, TNF- $\alpha$ increases PD-L1 expression in human breast cancer cells by promoting deubiquitination mediated by COP9 signalosome complex subunit 5 (CSN5) (12). When treated alone or in combination with IL-17, TNF- $\alpha$ upregulates PD-L1 expression in both human prostate cancer and colon cancer cells, mediated by the Akt/NF-kB and ERK/NF-KB pathways, respectively (13). In human renal cell carcinoma, TNF- $\alpha$ or IL-4 increases PD-L1 expression, accompanied by NF-KB or STAT6 activation, respectively. Combined treatment of the two has additive effects (14). IL- 6 has been reported to increase transcription of the PD-L1 gene by means of either JAK/STAT3 signaling in human prostate cancer cells (15) or MEK/ERK signaling in human lung cancer cells (16). IL-6 also increases PD-L1 expression at the post-transcriptional level by JAK1-mediated phosphorylation, which promotes glyco- sylation and stabilization of the PD-L1 protein in hepatocellular carcinoma cells (17). IL-27 can upregulate PD-L1 by means of STAT3 signaling in human ovarian cancer cell lines (18). Among anti-inflammatory cytokines, TGF $\beta$ was reported to increase PD-L1 expression in an Smad2-dependent manner in human lung cancer cells (19). Other than cytokines, stimulation of Toll-Like Receptor 4 (TLR4) by a lipopolysaccharide (LPS) induces PD-L1 expression in human bladder cancer cells via the ERK/ JNK pathway (20). In human neuroblastoma cells, PD-L1 can be upregulated in response to TLR3 stimulation, with simultaneous TLR9 ligation mitigating TLR3-mediated upregulation of PD-L1 (21).

Though PD-L1 expression is often represented by a tumor proportion score, which quantifies PD-L1 expression solely from viable tumor cells, accumulating evidence supports that a combined positive score, which integrates PD-L1 expression of tumor and non-tumor cells, is a more predictive biomarker (22), implying the significance of PD-L1 expressed by non-tumor cells in PD-1 blockade. Indeed, PD-L1 expressed by tumorinfiltrating immune cells promotes immune escape via diverse mechanisms (23). PD-L1 expressed on antigen-presenting cells (APCs), including dendritic cells and macrophages, delivers inhibitory signals during crosstalk with PD-1-expressing T cells or sequesters co-stimulatory molecule, CD80, in cis. Also, PD-L1 from activated T cells engages with PD-1 expressed on 


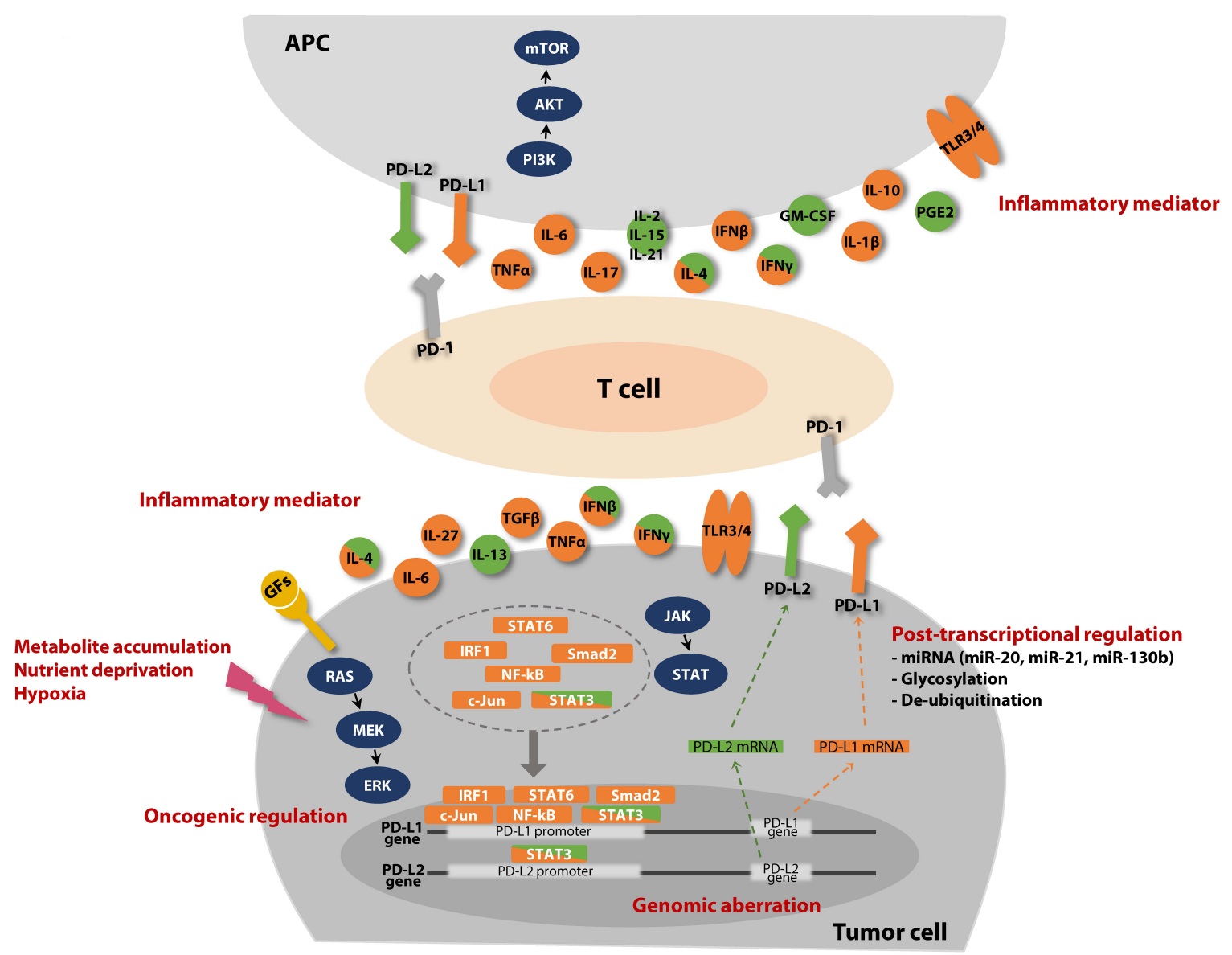

Fig. 2. Schematic overview of PD-L1 and PD-L2 positive-expression regulators in APCs (Up) or Tumor cells (Down). Molecules colored in orange or green regulate PD-L1 or PD-L2 expression, respectively. Molecules colored in orange and green regulate PD-L1 and PD-L2 expression at the same time.

other $\mathrm{T}$ cells or macrophages, the latter promoting M2 polarization. By the way, PD-L1 expressed on T cells can lead to an anergic state or apoptosis in activated T cells by acting as a receptor per se.

As in tumor cells, IFN- $\gamma$ induces PD-L1 in multiple types of immune cells, including monocytes $(24,25)$, monocytes-derived dendritic cells $(24,26)$, macrophages (26), and neutrophils (27). Although marginal, IFN- $\beta$ also increases PD-L1 expression in neutrophils (27) and dendritic cells (28). Among other pro-inflammatory cytokines, TNF- $\alpha$, IL-1 $\beta$, or IL- 6 upregulates PD-L1 expressed on monocyte-derived dendritic cells (29), whereas PD-L1 expressed on monocytes/macrophages is upregulated by TNF- $\alpha(30,31)$, IL-6 (32), or IL-17 (33). However, IL-17-induced upregulation of PD-L1 is indirectly mediated by several cytokines, particularly IL-10, produced by IL-17-activated monocytes. Another inflammatory cytokine, IL-12, regulates PD-L1 expressed on macrophages (34). Though IL-12 upregulates PD-L1 expression in monocyte-derived macrophages by means of increased IFN- $\gamma$ production, it also can downregulate PD-L1 expression in THP-1, a human monocytic cell line, presumably by decreased IL-10 production with inability to produce IFN- $\gamma$.

The role of anti-inflammatory cytokines in regulation of PD-L1 expression has also been investigated. As briefly mentioned above, IL-10 upregulates PD-L1 expression in monocytes (33, $35,36)$ and dendritic cells (37). Yet, IL-10-induced upregulation of PD-L1 is observed only in immature, monocyte-derived dendritic cells, not in LPS-matured one. The contradictory role of TGF $\beta$ in determining PD-L1 expression was observed, in that it upregulates PD-L1 expression in dendritic cells $(38,39)$, but downregulates it in monocytes (30). Also, TLR3 signaling induces PD-L1 expression in dendritic cells (40), and TLR4 signaling induces PD-L1 expression in monocytes (41), macrophages (42), and dendritic cells (43). Prostaglandin E2 (PGE2), which is a bioactive lipid that is closely connected with inflammation, is also involved in the induction of PD-L1 expression in myeloid cells, including macrophages and myeloid-de- 
rived suppressor cells (MDSCs) (44).

\section{PD-L2}

As a second ligand for PD-1, PD-L2 also endows PD-1 with an inhibitory function, although its mechanisms are not fully understood. Given that PD-L2 binds to PD-1 with a higher affinity than that of PD-L1 (45) and that its expression has been reported in many human malignancies, it is worth speculating about how its expression is regulated within the tumor microenvironment. PD-L2 expression was initially thought to be restricted to dendritic cells or macrophages, but recent studies reveal that PD-L2 expression is less restricted than previously thought. For example, a considerable proportion of peritoneal B1 B cells constitutively express PD-L2, which positivity enriches Phosphatidylcholine-specific B1 cells, as is crucial for innate defense against invading pathogens (46).

Like PD-L1, PD-L2 is upregulated by IFN- $\gamma$ treatment in tumor cells $(11,19,47,48)$. In line with how IFN- $\gamma$ orchestrates PD-L2 upregulation in tumor cells, IFN- $\beta$ has a similar effect via promoting STAT3 interaction with PD-L2 promoter (11). IL-4 and IL-13, $\mathrm{T}_{\mathrm{h}} 2$-type cytokines produced during Barrett's metaplasia, have been reported to induce PD-L2 expression in esophageal adenocarcinoma (49).

In immune cells, IFN- $\gamma$ and $\mathrm{T}_{\mathrm{h}} 2$ cytokines are involved in PD-L2 expression. IFN- $\gamma$ induces PD-L2 expression in human monocytes $(24,36,50)$, and IL-4 induces in murine macrophages $(42,51,52)$ and dendritic cells (51) in a STAT6-dependent manner. Yamazaki et al. also identified the granulocytemacrophage colony stimulating factor (GM-CSF) as an inducer of PD-L2 in both macrophages and dendritic cells, but its regulatory mechanism, which encompasses a transactivation effect of PU.1/ IRF4 and histone modification by PU.1/ p300, has been demonstrated recently (53). Additionally, common $\gamma$ chain cytokines, such as IL-2, IL-15, and IL-21, can induce PD-L2 expression in monocytes or macrophages (54). IL-10 also upregulates PD-L2 in monocytes (36), but downregulates PD-L2 in mature dendritic cells (24).

\section{T-CELL IMMUNORECEPTOR WITH IG AND ITIM DOMAINS (TIGIT) LIGANDS}

TIGIT is an inhibitory receptor, mainly expressed by NK cells, regulatory T cells, memory T cells, and exhausted CD8 T cells. When TIGIT was identified for the first time, it was also reported that human TIGIT can bind to three ligands, Poliovirus receptor (PVR, NECL5, CD155), PVR-related 2 (PVRL2, Nectin2, CD112), and PVR-related 3 (PVRL3, Nectin3, CD113), among which PVR has the highest affinity for TIGIT (55). PVR/ TIGIT engagement suppresses T-cell responses by phosphorylating immunoreceptor tyrosine-based inhibitory motif (ITIM) in the cytoplasmic tail of TIGIT or disturbing PVR/DNAXassociated molecule 1 (DNAM-1, CD226) engagement, which bolsters T-cell response.

PVR is an adhesion molecule frequently overexpressed in many types of solid and hematological malignancies, and its overexpression is associated with poor prognosis (56-59). Since PVR is a member of the Nectins and Nectin-like (Necl) family of molecules and is involved in various physiological processes, including cell-cell adhesion, movement, proliferation, and differentiation, its overexpression can have a protumorigenic effect in a manner intrinsic to tumor cells. In parallel, the oncogenic RAS/RAF/MEK/ERK signaling pathway upregulates PVR expression by means of direct binding of activator protein-1 (AP-1) to PVR promoter in mouse fibroblasts (60), a fact that is expected to be applicable to murine tumor cells. Despite the presence of the AP-1 binding sequence within the PVR promoter/enhancer in some human tumor cell lines (61), it is unclear if the RAS/RAF/MEK/ERK signaling increases PVR expression in human tumor cells as well. However, in terms of anti-tumor immune responses, whether PVR overexpression has a pro-tumorigenic or anti-tumorigenic role remains uncertain. In regulating the anti-tumor immune response, PVR can bind to three different receptorsDNAM-1, TIGIT, and CD96 - and contradictory effects can occur depending on the receptor that it binds to (62). Nevertheless, many previous studies present PVR as a stimulator of NK-cell function, emphasizing its interaction with activating receptor DNAM-1. For example, PVR can be induced as a part of an ATM/ATR-dependent DNA damage response ignited by either inherent genotoxic stress or genotoxic drug treatment in human multiple myeloma (MM). Particularly, when genotoxic stress imposed by nitric oxide and the related reactive nitrogen species induces PVR, the effect depends on transcriptional regulation by E2F1. Subsequently, induced PVR makes these tumors eliminable by means of DNAM-1 ligation in vitro (63, 64). Also, immuno-modulatory drugs targeting Cereblon, which breaks down transcriptional repressors of PVR, such as Ikaros family zinc finger protein-1 and -3 (IKZF1/3), also upregulate PVR expression and provoke NK-cell-mediated cytolysis in vitro (65). In terms of post-transcriptional regulation, when there is Endoplasmic reticulum (ER) stress, ER-associated degradationrelated molecule HRD1 increases and promotes PVR degradation in human hepatoma cell lines (66). In parallel, dysregulated small ubiquitin-like modifier (SUMO) conjugation, which results from SUMO-conjugating enzyme overexpression, also facilitates PVR degradation in human MM cell lines (67). Both studies demonstrated that diminished PVR expression caused tumor cells to evade recognition and elimination by NK cells. However, given that TIGIT binds to PVR with higher affinity than that of DNAM-1 $(55,68)$ and that TIGIT is highly expressed on tumor-infiltrating lymphocytes, how PVR expression on tumor cells affects effector cells in vivo needs further investigations.

Regarding tumor-infiltrating immune cells, it was reported that tumor-associated APCs represent a higher level of PVR than do circulating APCs, although factors responsible for upregulated PVR were not addressed in the study (69). However, since NF-kB signaling increases PVR expression on APCs upon 
TLR stimulation (70), an inflammatory microenvironment within tumor tissue may contribute to PVR upregulation in APCs (Fig. 1).

\section{T-CELL IMMUNOGLOBULIN DOMAIN AND MUCIN DOMAIN 3 (TIM-3) LIGANDS}

Tim-3, known as a hallmark of exhausted T cells, is one of the most commonly targeted checkpoints for immunotherapy. Four ligands have been described for Tim-3: Galectin-9 (Gal-9), Phosphatidylserine (PtdSer), High-mobility group box 1 (HMGB1), and Carcinoembryonic antigen-related cell adhesion molecule 1 (CEACAM1). Among them, Gal-9 and CEACAM1 have been reported to attenuate TCR signaling by dissociating HLA B-associated transcript 3 (BAT3) from Tim-3 $(71,72)$. Specifically, in the absence of the two ligands, Tim-3 is bound to BAT3, which interacts with Lymphocyte-specific protein tyrosine kinase (Lck). Since Lck-mediated phosphorylation of the TCR complex is critical for TCR downstream signaling, dissociation of BAT3 and subsequently, Lck upon Tim-3 ligation, impairs adjacent TCR downstream signaling and ultimately leads to apoptotic cell death. Furthermore, the intracellular interaction between Tim-3 and CEACAM1 supports maturation and surface trafficking of Tim-3. Therefore, the absence of CEACAM1 within T cells leads to intracellular accumulation of Tim-3 and its inability to interact with ligands, unleashing $\mathrm{T}$ cells from Tim-3mediated inhibition. On the other hand, PtdSer, which is exposed on the outer leaflet of apoptotic cells, triggers Tim-3 expressed on CD8 + dendritic cells or a subset of macrophages and induces phagocytosis; or extracellular HMGB1, a kind of alarmin, can be released into the tumor microenvironment and form a complex with free DNA. Since the formation of the complex assists internalization of DNA into dendritic cells to activate endosomal TLRs, sequestration of HMGB1 by Tim-3 can curtail the formation of the complex and the following dendritic cell activation. Until now, interaction between Tim-3 and PtdSer or HMGB1 has been known to affect T-cell function indirectly, but whether PtdSer or HMGB1 directly affects Tim-3-expressing T cells has to be evaluated.

Although expression of Gal-9 (73-75) or CEACAM1 (72, 76-78) in tumor cells and/or immune cells has been addressed, the factors implicated in their regulation have not been well explored. It has been reported that expression of Gal-9, the first ligand for Tim-3, is regulated by IFN (73, 74, 79). Unlike PD- 1 ligands, IFN- $\beta$ is a more robust regulator of Gal-9 expression in various cell lines than is IFN- $\gamma$. Moreover, Gal-9 has to be released extracellularly to serve as a ligand for Tim-3, and IFN- $\beta$ or IFN- $\gamma$ also enhances Gal- 9 secretion even in some of the tumor cells in which IFN- $\beta$ or IFN- $\gamma$ fails to increase Gal-9 expression or macrophages that possess constitutive Gal-9 expression (73). However, the other two studies delineated IFN- $\gamma$-mediated upregulation of Gal-9 in monocytes and granulocytic MDSCs, respectively, without covering the effect of IFN- $\beta$. Also, microRNA-dependent post-transcriptional regulation
$(80,81)$ and DNA (cytosine-5)-methyltransferase 3A (DNMT3A)mediated epigenetic modulation (82) of Gal-9 in tumor cells have been reported (Fig. 1).

\section{LYMPHOCYTE ACTIVATION GENE-3 (LAG-3, CD223) LIGANDS}

LAG-3 inhibits the anti-tumor immune response by synergizing with PD-1; a combination of anti-PD-1 and anti-LAG-3 is undergoing clinical trials. Notwithstanding the incomplete picture of molecular mechanisms, five ligands for LAG-3 have been discovered so far: MHC II, Galectin-3 (Gal-3), Liver sinusoidal endothelial cell lectin (LSECtin), $\alpha$-synuclein fibrils ( $\alpha$-syn), and Fibrinogen-like protein 1 (FGL1). Unlike other ligands, LAG-3 bound to $\alpha$-syn is associated with intercellular delivery of pathological $\alpha$-syn fibrils in the nervous system, which is irrelevant to immune response. Although it had been reported that LAG-3 can bind to MHC II with high affinity and regulate CD4 T cells by impeding interaction between MHC II and CD4, more recent studies have shown that discriminatory binding of LAG-3 to a stable MHC II/peptide complex (pMHC II) suppresses CD4 T cells by transducing inhibitory signals (83). Moreover, Maruhashi et al. also found that APCs that abundantly express stable pMHC II inhibit activation of CD8 T cells via LAG-3-dependent mechanism. In this regard, since MHC II transactivator (CIITA) can induce expression of MHC II accessory molecules, which are involved in pMHC II formation, as well as that of MHC II, IFN- $\gamma$-mediated upregulation of CIITA expression may increase pMHC II in various types of cells, including certain tumor cells (84). Alternatively, other ligands, Gal-3 (85), LSECtin (86), and FGL1 (87), have been reported to engage with LAG-3 to negatively regulate CD8 T cells in the tumor microenvironment. Even though Kouo et al. demonstrated that intratumoral CD8 T cells and stromal cells are major sources of Gal-3 (85), Gal-3 is also secreted by many types of tumor cells (88). It is reported that Gal-3 is exocytosed from stromal cells and endocytosed by tumor cells in pre-B cell lymphoma. Afterwards, internalized Gal-3 auto-activates Gal-3 transcription accompanied by NK-кB signaling in tumor cells (89). Though the study rarely provides direct evidence for causality between NF-KB and Gal-3, it is conceivable that increased Gal-3 transcription is a result of NF- $\mathrm{\kappa B}$ activation, taking into account the NF-אB-mediated Gal-3 expression in other types of cells $(90,91)$. LSECtin or FGL1 is expressed in the liver under normal physiological conditions and is highly upregulated in some tumor cells (86). Although LSECtin is induced by IL-6 or IL-10 treatment in tumor cells, it can also be induced in human monocyte-derived dendritic cells by IL-4 treatment (92). IL-6 also increases FGL1 in human hepatoma cells (93). Future studies are required to verify the precise molecular mechanisms of these ligands and the possibility of their cooperation in LAG-3-mediated T cell inhibition (Fig. 1). 


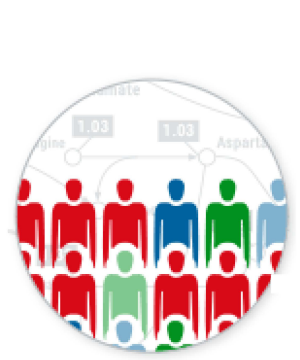

Individual difference

1. Tumor microenvironment

2. TME-derived factors

3. Signaling network

4. Immune checkpoint ligand expression

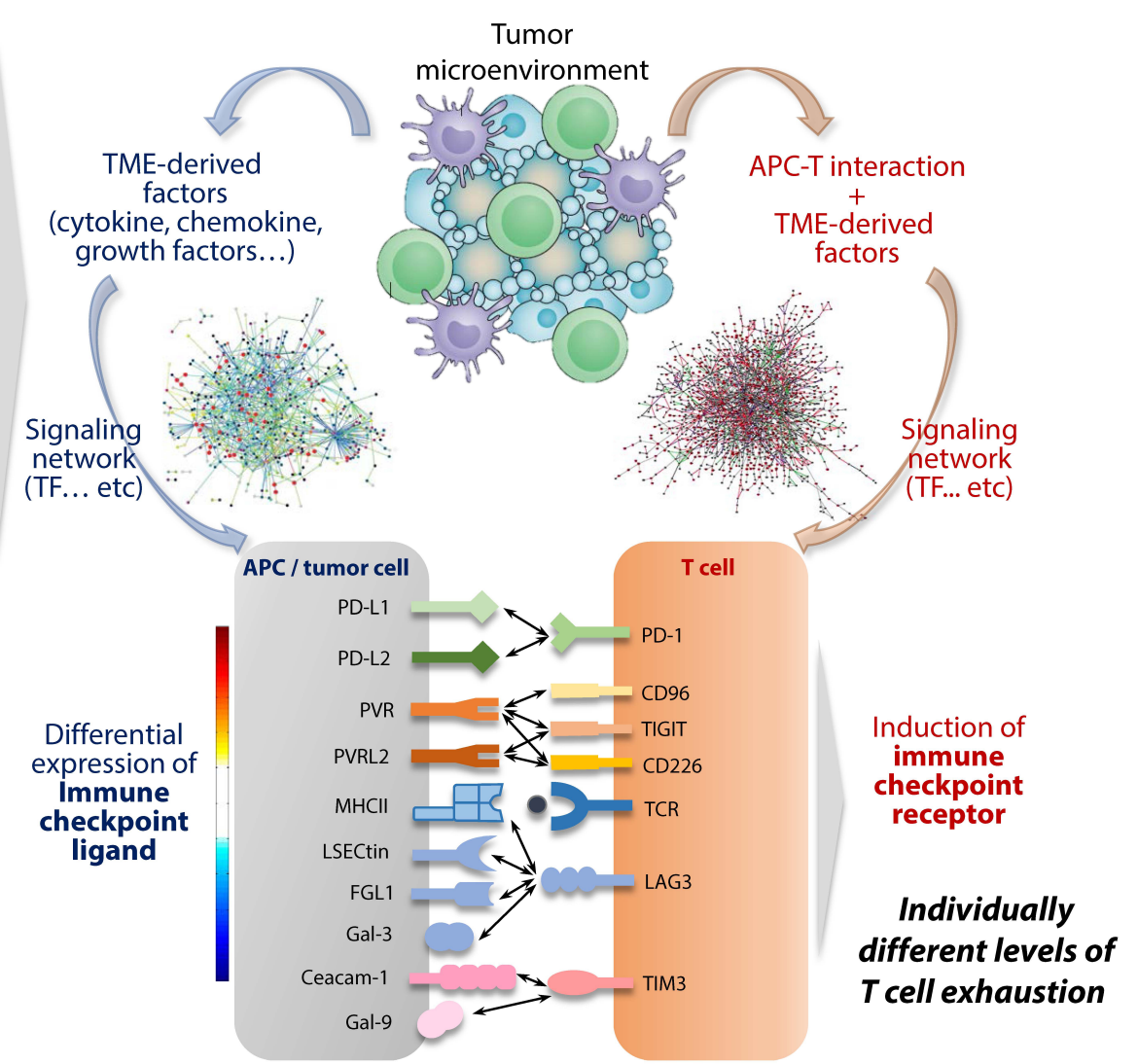

Tumor

microenvironment -derived growth factors...)

Signaling

network

nduction of

immune

checkpoint

Individually

different levels of

cell exhaustion

Fig. 3. ICRs are co-regulated and simultaneously expressed by common factors. In contrast, Individual difference in TME-derived factors or signaling network induce diverse patterns of ICLs. Heterogeneity in expression of ICLs can afford patient stratification for customized ICB therapy.

\section{CONCLUSIONS}

In the tumor microenvironment, tumor cells build a permissive environment for growth, executing distinct strategies. As a tumor progresses, ICRs are simultaneously induced by common factors, whereas the expression of the corresponding ICLs has overlapping but independent regulation. Further, there are diverse regulators in the tumor microenvironment, each of which may have inconsistent effects on ICL expression depending on the cellular context, creating individually different expressions of ICLs. Because ICL expression patterns could be of relevance to indicate the activity of immune checkpoint pathways, differential expression profiles of ICLs among individuals can be used to predict the treatment response of the immune checkpoint blockade. For instance, many studies have shown that PD-L1 expression is associated with superior response to PD-1 blockade (94). Our previous study demonstrated that the complementary expression patterns of PVR and PD-L1 are key determinants for PD-1 blockade (95). Another study revealed that $\mathrm{CD} 276$, which is an incompletely elucidated ICL, could be used as potential biomarker for PD-1 blockade (96). By querying a set of verified ICLs and identifying robust ICL-based biomarkers, it is possible to achieve a higher responder rate and tailor precision immunotherapies. (Fig. 3).

\section{ACKNOWLEDGEMENTS}

This study was supported by the National Research Foundation of Korea (NRF) grant funded by the Korea government (MSIT) (2017R1A5A1014560, 2019M3A9B6065221) and by the National Institute of Biological Resources funded by the Ministry of Environment (MOE) (NIBR202122202). This study was also supported by the Korean Health Technology R\&D Project (HV20C0144) through the Korean Health Industry Development Institute (KHIDI) funded by the Ministry of Health \& Welfare. The funder had no role in study design, data collection and analysis, decision to publish, or preparation of the manuscript. 


\section{CONFLICTS OF INTEREST}

The authors have no conflicting interests.

\section{REFERENCES}

1. Patsoukis N, Wang Q, Strauss L and Boussiotis VA (2020) Revisiting the PD-1 pathway. Sci Adv 6, eabd2712

2. Marin-Acevedo JA, Kimbrough EO and Lou Y (2021) Next generation of immune checkpoint inhibitors and beyond. J Hematol Oncol 14, 45

3. Anderson AC, Joller N and Kuchroo VK (2016) Lag-3, Tim-3, and TIGIT: co-inhibitory receptors with specialized functions in immune regulation. Immunity 44, 989-1004

4. Chihara N, Madi A, Kondo T et al (2018) Induction and transcriptional regulation of the co-inhibitory gene module in T cells. Nature 558, 454-459

5. Yadollahi $\mathrm{P}$, Jeon YK, Ng WL and Choi I (2021) Current understanding of cancer-intrinsic PD-L1: regulation of expression and its protumoral activity. BMB Rep 54, 12-20

6. Yu Y, Liang Y, Li D et al (2021) Glucose metabolism involved in PD-L1-mediated immune escape in the malignant kidney tumour microenvironment. Cell Death Discov 7, 15

7. Byun JK, Park M, Lee S et al (2020) Inhibition of glutamine utilization synergizes with immune checkpoint inhibitor to promote antitumor immunity. Mol Cell 80, 592606 e598

8. Feng J, Yang H, Zhang Y et al (2017) Tumor cell-derived lactate induces TAZ-dependent upregulation of PD-L1 through GPR81 in human lung cancer cells. Oncogene 36, 5829-5839

9. Barsoum IB, Smallwood CA, Siemens DR and Graham $\mathrm{CH}$ (2014) A mechanism of hypoxia-mediated escape from adaptive immunity in cancer cells. Cancer Res 74, 665-674

10. Dong H, Strome SE, Salomao DR et al (2002) Tumorassociated $\mathrm{B} 7-\mathrm{H} 1$ promotes T-cell apoptosis: a potential mechanism of immune evasion. Nat Med 8, 793-800

11. Garcia-Diaz A, Shin DS, Moreno BH et al (2017) interferon receptor signaling pathways regulating PD-L1 and PD-L2 expression. Cell Rep 19, 1189-1201

12. Lim SO, Li CW, Xia W et al (2016) Deubiquitination and stabilization of PD-L1 by CSN5. Cancer Cell 30, 925-939

13. Wang $X$, Yang L, Huang $F$ et al (2017) Inflammatory cytokines IL-17 and TNF-alpha up-regulate PD-L1 expression in human prostate and colon cancer cells. Immunol Lett 184, 7-14

14. Quandt D, Jasinski-Bergner S, Muller U, Schulze B and Seliger B (2014) Synergistic effects of IL-4 and TNFalpha on the induction of $\mathrm{B} 7-\mathrm{H} 1$ in renal cell carcinoma cells inhibiting allogeneic T cell proliferation. J Transl Med 12, 151

15. Xu L, Chen X, Shen M et al (2018) Inhibition of IL-6JAK/Stat3 signaling in castration-resistant prostate cancer cells enhances the NK cell-mediated cytotoxicity via alteration of PD-L1/NKG2D ligand levels. Mol Oncol 12, 269-286

16. Shen MJ, Xu LJ, Yang $L$ et al (2017) Radiation alters
PD-L1/NKG2D ligand levels in lung cancer cells and leads to immune escape from NK cell cytotoxicity via IL-6-MEK/Erk signaling pathway. Oncotarget 8, 80506-80520

17. Chan LC, Li CW, Xia W et al (2019) IL-6/JAK1 pathway drives PD-L1 Y112 phosphorylation to promote cancer immune evasion. J Clin Invest 129, 3324-3338

18. Carbotti G, Barisione G, Airoldi I et al (2015) IL-27 induces the expression of IDO and PD-L1 in human cancer cells. Oncotarget 6, 43267-43280

19. David JM, Dominguez C, McCampbell KK, Gulley JL, Schlom J and Palena C (2017) A novel bifunctional antiPD-L1/TGF-beta Trap fusion protein (M7824) efficiently reverts mesenchymalization of human lung cancer cells. Oncoimmunology 6, e1349589

20. Qian Y, Deng J, Geng L et al (2008) TLR4 signaling induces $\mathrm{B} 7-\mathrm{H} 1$ expression through MAPK pathways in bladder cancer cells. Cancer Invest 26, 816-821

21. Boes M and Meyer-Wentrup F (2015) TLR3 triggering regulates PD-L1 (CD274) expression in human neuroblastoma cells. Cancer Lett 361, 49-56

22. Yamashita K, Iwatsuki M, Harada K et al (2020) Prognostic impacts of the combined positive score and the tumor proportion score for programmed death ligand-1 expression by double immunohistochemical staining in patients with advanced gastric cancer. Gastric Cancer 23, 95-104

23. Shklovskaya E and Rizos H (2020) Spatial and temporal changes in pd-I1 expression in cancer: the role of genetic drivers, tumor microenvironment and resistance to therapy. Int J Mol Sci 21, 7139

24. Brown JA, Dorfman DM, Ma FR et al (2003) Blockade of programmed death-1 ligands on dendritic cells enhances T cell activation and cytokine production. J Immunol 170, 1257-1266

25. Muthumani K, Shedlock DJ, Choo DK et al (2011) HIV-mediated phosphatidylinositol 3-kinase/serine-threonine kinase activation in APCs leads to programmed death-1 ligand upregulation and suppression of HIV-specific CD8 T cells. J Immunol 187, 2932-2943

26. Kryczek I, Wei S, Gong W et al (2008) Cutting edge: IFN-gamma enables APC to promote memory Th17 and abate Th1 cell development. J Immunol 181, 5842-5846

27. de Kleijn S, Langereis JD, Leentjens J et al (2013) IFN-gamma-stimulated neutrophils suppress lymphocyte proliferation through expression of PD-L1. PLoS One 8, e72249

28. Schreiner B, Mitsdoerffer M, Kieseier BC et al (2004) Interferon-beta enhances monocyte and dendritic cell expression of B7-H1 (PD-L1), a strong inhibitor of autologous T-cell activation: relevance for the immune modulatory effect in multiple sclerosis. J Neuroimmunol 155, 172-182

29. Karakhanova S, Meisel S, Ring S, Mahnke K and Enk AH (2010) ERK/p38 MAP-kinases and PI3K are involved in the differential regulation of $\mathrm{B} 7-\mathrm{H} 1$ expression in DC subsets. Eur J Immunol 40, 254-266

30. Ou JN, Wiedeman AE and Stevens AM (2012) TNF-alpha and TGF-beta counter-regulate PD-L1 expression on monocytes in systemic lupus erythematosus. Sci Rep 2, 295

31. Hartley G, Regan D, Guth A and Dow S (2017) Regulation of PD-L1 expression on murine tumor-associated 
monocytes and macrophages by locally produced TNFalpha. Cancer Immunol Immunother 66, 523-535

32. Zhang W, Liu Y, Yan Z et al (2020) IL-6 promotes PD-L1 expression in monocytes and macrophages by decreasing protein tyrosine phosphatase receptor type $\mathrm{O}$ expression in human hepatocellular carcinoma. J Immunother Cancer 8

33. Zhao Q, Xiao X, Wu Y et al (2011) Interleukin-17educated monocytes suppress cytotoxic T-cell function through $\mathrm{B} 7-\mathrm{H} 1$ in hepatocellular carcinoma patients. Eur J Immunol 41, 2314-2322

34. Xiong HY, Ma TT, Wu BT, Lin Y and Tu ZG (2014) IL-12 regulates $\mathrm{B} 7-\mathrm{H} 1$ expression in ovarian cancer-associated macrophages by effects on NF-kappaB signalling. Asian Pac J Cancer Prev 15, 5767-5772

35. Jiang C, Yuan F, Wang J and Wu L (2017) Oral squamous cell carcinoma suppressed antitumor immunity through induction of PD-L1 expression on tumor-associated macrophages. Immunobiology 222, 651-657

36. Taube JM, Young GD, McMiller TL et al (2015) Differential expression of immune-regulatory genes associated with PD-L1 display in melanoma: implications for PD-1 pathway blockade. Clin Cancer Res 21, 3969-3976

37. Curiel TJ, Wei S, Dong H et al (2003) Blockade of B7-H1 improves myeloid dendritic cell-mediated antitumor immunity. Nat Med 9, 562-567

38. Song S, Yuan P, Wu H et al (2014) Dendritic cells with an increased PD-L1 by TGF-beta induce T cell anergy for the cytotoxicity of hepatocellular carcinoma cells. Int Immunopharmacol 20, 117-123

39. Ni XY, Sui HX, Liu Y, Ke SZ, Wang YN and Gao FG (2012) TGF-beta of lung cancer microenvironment upregulates $\mathrm{B} 7 \mathrm{H} 1$ and GITRL expression in dendritic cells and is associated with regulatory $\mathrm{T}$ cell generation. Oncol Rep 28, 615-621

40. Pulko V, Liu X, Krco CJ et al (2009) TLR3-stimulated dendritic cells up-regulate B7-H1 expression and influence the magnitude of CD8 $\mathrm{T}$ cell responses to tumor vaccination. J Immunol 183, 3634-3641

41. Huang G, Wen Q, Zhao Y, Gao Q and Bai Y (2013) NF-kappaB plays a key role in inducing CD274 expression in human monocytes after lipopolysaccharide treatment. PLoS One 8, e61602

42. Loke P and Allison JP (2003) PD-L1 and PD-L2 are differentially regulated by Th1 and Th2 cells. Proc Natl Acad Sci U S A 100, 5336-5341

43. Mezzadra R, Sun C, Jae LT et al (2017) Identification of CMTM6 and CMTM4 as PD-L1 protein regulators. Nature 549, 106-110

44. Prima V, Kaliberova LN, Kaliberov S, Curiel DT and Kusmartsev S (2017) COX2/mPGES1/PGE2 pathway regulates PD-L1 expression in tumor-associated macrophages and myeloid-derived suppressor cells. Proc Natl Acad Sci U S A 114, 1117-1122

45. Youngnak $\mathrm{P}$, Kozono $\mathrm{Y}$, Kozono $\mathrm{H}$ et al (2003) Differential binding properties of $\mathrm{B} 7-\mathrm{H} 1$ and $\mathrm{B} 7-\mathrm{DC}$ to programmed death-1. Biochem Biophys Res Commun 307, 672-677

46. Zhong X, Tumang JR, Gao W, Bai C and Rothstein TL (2007) PD-L2 expression extends beyond dendritic cells/macrophages to B1 cells enriched for $\mathrm{V}(\mathrm{H}) 11 / \mathrm{V}(\mathrm{H}) 12$ and phosphatidylcholine binding. Eur J Immunol 37, 2405-2410

47. Wang $\mathrm{H}$, Yao $\mathrm{H}$, Li C et al (2017) PD-L2 expression in colorectal cancer: Independent prognostic effect and targetability by deglycosylation. Oncoimmunology 6 , e1327494

48. Fu Y, Liu CJ, Kobayashi DK et al (2020) GATA2 regulates constitutive PD-L1 and PD-L2 expression in brain tumors. Sci Rep 10, 9027

49. Derks S, Nason KS, Liao X et al (2015) Epithelial PD-L2 expression marks Barrett's esophagus and esophageal adenocarcinoma. Cancer Immunol Res 3, 1123-1129

50. Latchman Y, Wood CR, Chernova T et al (2001) PD-L2 is a second ligand for PD-1 and inhibits $\mathrm{T}$ cell activation. Nat Immunol 2, 261-268

51. Yamazaki T, Akiba $\mathrm{H}$, Iwai $\mathrm{H}$ et al (2002) Expression of programmed death 1 ligands by murine T cells and APC. J Immunol 169, 5538-5545

52. Huber S, Hoffmann $R$, Muskens $F$ and Voehringer $D$ (2010) Alternatively activated macrophages inhibit T-cell proliferation by Stat6-dependent expression of PD-L2. Blood 116, 3311-3320

53. Inaba K, Yashiro T, Hiroki I, Watanabe R, Kasakura K and Nishiyama C (2020) Dual roles of PU.1 in the expression of PD-L2: direct transactivation with IRF4 and indirect epigenetic regulation. J Immunol 205, 822-829

54. Kinter AL, Godbout EJ, McNally JP et al (2008) The common gamma-chain cytokines IL-2, IL-7, IL-15, and IL-21 induce the expression of programmed death- 1 and its ligands. J Immunol 181, 6738-6746

55. Yu X, Harden K, Gonzalez LC et al (2009) The surface protein TIGIT suppresses $\mathrm{T}$ cell activation by promoting the generation of mature immunoregulatory dendritic cells. Nat Immunol 10, 48-57

56. Masson D, Jarry A, Baury B et al (2001) Overexpression of the CD155 gene in human colorectal carcinoma. Gut 49, 236-240

57. Nakai R, Maniwa Y, Tanaka Y et al (2010) Overexpression of Necl-5 correlates with unfavorable prognosis in patients with lung adenocarcinoma. Cancer Sci 101, 1326-1330

58. Bevelacqua V, Bevelacqua Y, Candido S et al (2012) Nectin like- 5 overexpression correlates with the malignant phenotype in cutaneous melanoma. Oncotarget 3, 882-892

59. Triki H, Charfi S, Bouzidi L et al (2019) CD155 expression in human breast cancer: clinical significance and relevance to natural killer cell infiltration. Life Sci 231, 116543

60. Hirota T, Irie K, Okamoto R, Ikeda W and Takai Y (2005) Transcriptional activation of the mouse Necl-5/Tage4/PVR/ CD155 gene by fibroblast growth factor or oncogenic Ras through the Raf-MEK-ERK-AP-1 pathway. Oncogene 24, 2229-2235

61. Schummer P, Kuphal S, Vardimon L, Bosserhoff AK and Kappelmann M (2016) Specific c-Jun target genes in malignant melanoma. Cancer Biol Ther 17, 486-497

62. Martinet $L$ and Smyth MJ (2015) Balancing natural killer cell activation through paired receptors. Nat Rev Immunol 15, 243-254

63. Fionda C, Abruzzese MP, Zingoni A et al (2015) Nitric oxide donors increase PVR/CD155 DNAM-1 ligand express- 
ion in multiple myeloma cells: role of DNA damage response activation. BMC Cancer 15, 17

64. Soriani A, Zingoni A, Cerboni C et al (2009) ATM-ATRdependent up-regulation of DNAM-1 and NKG2D ligands on multiple myeloma cells by therapeutic agents results in enhanced NK-cell susceptibility and is associated with a senescent phenotype. Blood 113, 3503-3511

65. Fionda C, Abruzzese MP, Zingoni A et al (2015) The IMiDs targets IKZF-1/3 and IRF4 as novel negative regulators of NK cell-activating ligands expression in multiple myeloma. Oncotarget 6, 23609-23630

66. Gong J, Fang L, Liu R et al (2014) UPR decreases CD226 ligand CD155 expression and sensitivity to NK cell-mediated cytotoxicity in hepatoma cells. Eur J Immunol 44, 3758-3767

67. Zitti B, Molfetta R, Fionda C et al (2017) Innate immune activating ligand SUMOylation affects tumor cell recognition by NK cells. Sci Rep 7, 10445

68. Stanietsky N, Rovis TL, Glasner A et al (2013) Mouse TIGIT inhibits NK-cell cytotoxicity upon interaction with PVR. Eur J Immunol 43, 2138-2150

69. Chauvin JM, Pagliano O, Fourcade J et al (2015) TIGIT and PD-1 impair tumor antigen-specific CD8(+) T cells in melanoma patients. J Clin Invest 125, 2046-2058

70. Kamran N, Takai Y, Miyoshi J, Biswas SK, Wong JS and Gasser S (2013) Toll-like receptor ligands induce expression of the costimulatory molecule CD155 on antigen-presenting cells. PLoS One 8, e54406

71. Rangachari M, Zhu C, Sakuishi K et al (2012) Bat3 promotes $\mathrm{T}$ cell responses and autoimmunity by repressing Tim-3-mediated cell death and exhaustion. Nat Med $18,1394-1400$

72. Huang $\mathrm{YH}$, Zhu C, Kondo $\mathrm{Y}$ et al (2015) CEACAM1 regulates TIM-3-mediated tolerance and exhaustion. Nature 517, 386-390

73. Yang R, Sun L, Li CF et al (2021) Galectin-9 interacts with PD-1 and TIM-3 to regulate T cell death and is a target for cancer immunotherapy. Nat Commun 12, 832

74. Li H, Wu K, Tao K et al (2012) Tim-3/galectin-9 signaling pathway mediates T-cell dysfunction and predicts poor prognosis in patients with hepatitis B virus-associated hepatocellular carcinoma. Hepatology 56, 1342-1351

75. Wiener Z, Kohalmi B, Pocza P et al (2007) TIM-3 is expressed in melanoma cells and is upregulated in TGF-beta stimulated mast cells. J Invest Dermatol 127, 906-914

76. Kammerer R, Stober D, Singer BB, Obrink B and Reimann J (2001) Carcinoembryonic antigen-related cell adhesion molecule 1 on murine dendritic cells is a potent regulator of T cell stimulation. J Immunol 166, 6537-6544

77. Horst AK, Bickert T, Brewig N et al (2009) CEACAM1+ myeloid cells control angiogenesis in inflammation. Blood $113,6726-6736$

78. Gebauer F, Wicklein D, Horst J et al (2014) Carcinoembryonic antigen-related cell adhesion molecules (CEACAM) 1,5 and 6 as biomarkers in pancreatic cancer. PLoS One 9, e113023

79. Dardalhon V, Anderson AC, Karman J et al (2010) Tim-3/ galectin-9 pathway: regulation of Th1 immunity through promotion of CD11b+Ly-6G + myeloid cells. J Immunol $185,1383-1392$

80. Zhou J, Jiang Y, Zhang H et al (2019) Clinicopathological implications of $\operatorname{TIM} 3(+)$ tumor-infiltrating lymphocytes and the miR-455-5p/Galectin-9 axis in skull base chordoma patients. Cancer Immunol Immunother 68, 1157-1169

81. Yang $Q$, Jiang $W$, Zhuang $C$ et al (2015) microRNA-22 downregulation of galectin-9 influences lymphocyte apoptosis and tumor cell proliferation in liver cancer. Oncol Rep 34, 1771-1778

82. Zhang L, Tian S, Zhao M et al (2020) SUV39H1-DNMT3Amediated epigenetic regulation of Tim-3 and galectin-9 in the cervical cancer. Cancer Cell Int 20, 325

83. Maruhashi T, Okazaki IM, Sugiura D et al (2018) LAG-3 inhibits the activation of $\mathrm{CD} 4(+) \mathrm{T}$ cells that recognize stable pMHCII through its conformation-dependent recognition of pMHCII. Nat Immunol 19, 1415-1426

84. Reith W, LeibundGut-Landmann S and Waldburger JM (2005) Regulation of MHC class II gene expression by the class II transactivator. Nat Rev Immunol 5, 793-806

85. Kouo T, Huang L, Pucsek AB et al (2015) Galectin-3 shapes antitumor immune responses by suppressing CD8 + T cells via LAG-3 and inhibiting expansion of plasmacytoid dendritic cells. Cancer Immunol Res 3, 412-423

86. Xu F, Liu J, Liu D et al (2014) LSECtin expressed on melanoma cells promotes tumor progression by inhibiting antitumor T-cell responses. Cancer Res 74, 3418-3428

87. Wang J, Sanmamed MF, Datar I et al (2019) Fibrinogenlike Protein 1 Is a Major Immune Inhibitory Ligand of LAG-3. Cell 176, 334-347 e312

88. Nakahara S, Oka N and Raz A (2005) On the role of galectin-3 in cancer apoptosis. Apoptosis 10, 267-275

89. Fei F, Joo EJ, Tarighat SS et al (2015) B-cell precursor acute lymphoblastic leukemia and stromal cells communicate through Galectin-3. Oncotarget 6, 11378-11394

90. Kim K, Mayer EP and Nachtigal M (2003) Galectin-3 expression in macrophages is signaled by Ras/MAP kinase pathway and up-regulated by modified lipoproteins. Biochim Biophys Acta 1641, 13-23

91. Liu L, Sakai T, Sano N and Fukui K (2004) Nucling mediates apoptosis by inhibiting expression of galectin-3 through interference with nuclear factor kappaB signalling. Biochem J 380, 31-41

92. Dominguez-Soto A, Aragoneses-Fenoll L, Martin-Gayo E et al (2007) The DC-SIGN-related lectin LSECtin mediates antigen capture and pathogen binding by human myeloid cells. Blood 109, 5337-5345

93. Liu Z and Ukomadu C (2008) Fibrinogen-like protein 1, a hepatocyte derived protein is an acute phase reactant. Biochem Biophys Res Commun 365, 729-734

94. Doroshow DB, Bhalla S, Beasley MB et al (2021) PD-L1 as a biomarker of response to immune-checkpoint inhibitors. Nat Rev Clin Oncol 18, 345-362

95. Lee BR, Chae S, Moon J et al (2020) Combination of PD-L1 and PVR determines sensitivity to PD-1 blockade. JCl Insight 5, e128633

96. Yonesaka K, Haratani K, Takamura S et al (2018) B7-H3 negatively modulates ctl-mediated cancer immunity. Clin Cancer Res 24, 2653-2664 